ANNALES

POLONICI MATHEMATICI

$96.1(2009)$

\title{
On uniqueness of meromorphic functions sharing three values and a set consisting of two small meromorphic functions
}

\author{
by XiaO-Min Li (Qingdao) and Hong-Xun Yi (Jinan)
}

\begin{abstract}
We deal with a uniqueness theorem of two meromorphic functions that share three values with weights and also share a set consisting of two small meromorphic functions. Our results improve those by G. Brosch, I. Lahiri \& P. Sahoo, T. C. Alzahary \& H. X. Yi, P. Li \& C. C. Yang, and others.
\end{abstract}

1. Introduction and main results. In this paper, by meromorphic functions we will always mean meromorphic functions in the complex plane. We adopt the standard notations of the Nevanlinna theory of meromorphic functions as explained in [6], [10] and [15]. It will be convenient to let $E$ denote any set of positive real numbers of finite linear measure, not necessarily the same at each occurrence. For a nonconstant meromorphic function $h$, we denote by $T(r, h)$ the Nevanlinna characteristic of $h$ and by $S(r, h)$ any quantity satisfying

$$
S(r, h)=o(T(r, h)) \quad(r \rightarrow \infty, r \notin E) .
$$

Let $f(z)$ and $g(z)$ be two nonconstant meromorphic functions, and let $a$ be a value in the extended plane. We say that $f$ and $g$ share the value a $C M$ provided that $f$ and $g$ have the same $a$-points with the same multiplicities. Similarly, we say that $f$ and $g$ share the value a IM provided that $f$ and $g$ have the same $a$-points ignoring multiplicities (see [15]). We say that $a$ is a small function of $f$ if $a$ is a meromorphic function satisfying $T(r, a)=S(r, f)$ (see [15]). If $a$ is a small function such that $\bar{N}(r, 1 /(f-a))=S(r, f)$, then we say that $a$ is an exceptional small function of $f$ (see [11]). If $\alpha_{1}, \alpha_{2}, \alpha_{3}$, $\alpha_{4}$ are four small functions of $g$ such that $f=\left(\alpha_{1} g+\alpha_{2}\right) /\left(\alpha_{3} g+\alpha_{4}\right)$, where $\alpha_{1} \alpha_{4}-\alpha_{2} \alpha_{3} \not \equiv 0$, then $f$ is said to be a quasi-Möbius transformation of $g$ (see [15]). We also need the following definition.

2000 Mathematics Subject Classification: Primary 30D35, 30D30.

Key words and phrases: meromorphic functions, weighted sharing of values, sharing sets of small meromorphic functions, uniqueness theorems. 
Definition 1.1 (see [1, Definition 1]). Let $p$ be a positive integer and $a \in \mathbb{C} \cup\{\infty\}$. Then we denote by $N_{p)}(r, 1 /(f-a))$ the counting function of those zeros of $f-a$ (counted with proper multiplicities) whose multiplicities are not greater than $p$, and by $\bar{N}_{p)}(r, 1 /(f-a))$ the corresponding reduced counting function (ignoring multiplicities). By $N_{(p}(r, 1 /(f-a))$ we denote the counting function of those zeros of $f-a$ (counted with proper multiplicities) whose multiplicities are not less than $p$, and by $\bar{N}_{(p}(r, 1 /(f-a))$ the corresponding reduced counting function (ignoring multiplicities).

Let $f$ and $g$ be two nonconstant meromorphic functions, and let $a$ be a value in the extended plane. Let $S$ be a subset of distinct elements in the extended plane. We define $E_{f}(S)=\bigcup_{a \in S}\{z: f(z)=a\}$, where each $a$-point of $f$ with multiplicity $m$ is repeated $m$ times in $E_{f}(S)$ (see [4]). Similarly, we define $\bar{E}_{f}(S)=\bigcup_{a \in S}\{z: f(z)=a\}$, where each point in $\bar{E}_{f}(a)$ is counted only once. We say that $f$ and $g$ share the set $S C M$ provided $E_{f}(S)=E_{g}(S)$. We say that $f$ and $g$ share the set $S I M$ provided $\bar{E}_{f}(S)=\bar{E}_{g}(S)$. Below, the notation $f=a \Rightarrow g=a$ means $\bar{E}_{f}(\{a\}) \subseteq \bar{E}_{g}(\{a\})$. If $S$ is a set consisting of small meromorphic functions of $f$ and $g$, then the above definitions have the same meanings.

In 1989, G. Brosch proved the following theorem.

Theorem A (see [3]). Let $f$ and $g$ be two distinct nonconstant meromorphic functions such that $f$ and $g$ share $0,1, \infty C M$, and let $a$ and $b$ be two distinct finite complex numbers such that $a, b \notin\{0,1\}$. If $f-a$ and $g-b$ share $0 I M$, then $f$ is a Möbius transformation of $g$.

Regarding Theorem A, it is natural to ask the following two questions.

QUESTION 1.1 (see [8])). Is it possible to relax in any way the nature of sharing any one of the values 0,1 and $\infty$ in Theorem A?

Question 1.2. What can be said if the two distinct finite complex numbers $a(\neq 0,1)$ and $b(\neq 0,1)$ are replaced with two small meromorphic functions $a(\not \equiv 0,1, \infty)$ and $b(\not \equiv 0,1, \infty)$ respectively?

In this paper, we will deal with these two questions. To this end we employ the idea of weighted sharing of values which measures how close a shared value is to being shared IM or to being shared CM. The notion is explained in the following definition.

Definition 1.2 (see [7, Definition 4]). Let $k$ be a nonnegative integer or infinity. For any $a \in \mathbb{C} \cup\{\infty\}$, we denote by $E_{k}(a, f)$ the set of all $a$-points of $f$, where an $a$-point of multiplicity $m$ is counted $m$ times if $m \leq k$, and $k+1$ times if $m>k$. If $E_{k}(a, f)=E_{k}(a, g)$, we say that $f, g$ share the value $a$ with weight $k$. 
REMARK 1.1. Definition 1.2 implies that if $f, g$ share a value $a$ with weight $k$, then $z_{0}$ is a zero of $f-a$ with multiplicity $m(\leq k)$ if and only if it is a zero of $g-a$ with multiplicity $m(\leq k)$, and $z_{0}$ is a zero of $f-a$ with multiplicity $m(>k)$ if and only if it is a zero of $g-a$ with multiplicity $n(>k)$, where $m$ is not necessarily equal to $n$. Throughout this paper, we write $f, g$ share $(a, k)$ to mean that $f, g$ share the value $a$ with weight $k$. Clearly, if $f, g$ share $(a, k)$, then $f, g$ share $(a, p)$ for all integer $p, 0 \leq p<k$. Also we note that $f, g$ share a value $a$ IM or CM if and only if $f, g$ share $(a, 0)$ or $(a, \infty)$, respectively.

Recently, I. Lahiri and P. Sahoo proved the following theorem which improves Theorem A and Theorem 1 in [2], and deals with Question 1.1.

Theorem B (see [9, Theorem 1.1]). Let $f$ and $g$ be two distinct nonconstant meromorphic functions such that $f$ and $g$ share $\left(a_{1}, 1\right),\left(a_{2}, m\right)$ and $\left(a_{3}, k\right)$, where $\left\{a_{1}, a_{2}, a_{3}\right\}=\{0,1, \infty\}$, and $m$ and $k$ are two positive integers satisfying $(m-1)(m k-1)>(1+m)^{2}$, and let $a$ and $b$ be two distinct finite complex numbers such that $a, b \notin\{0,1\}$. If $f-a$ and $g-b$ share 0 IM, then $f$ and $g$ share 0,1 and $\infty C M$, and $f-a$ and $g-b$ share $0 C M$. Moreover, $f$ and $g$ satisfy one of the following nine relations:

(i) $f g=1$ with $a b=1$;

(vi) $f=(1-a) g+a$ with $a b=a+b$;

(ii) $f+g=1$ with $a+b=1$;

(vii) $f=\frac{(1-a) g}{1-b}+\frac{b-a}{b-1}$;

(iii) $f=\frac{g}{g-1}$ with $a b=a+b$;

(viii) $f=\frac{a g}{g+a-1}$ with $a+b=1$;

(iv) $f=a g$ with $a b=1$;

(ix) $f=\frac{a(b-1) g}{(b-a) g+(a-1) b}$.

(v) $f=\frac{a g}{b}$;

In 1997, P. Li and C. C. Yang proved the following result dealing with Question 1.2.

Theorem C (see [11, Theorem 6]). Let $f$ and $g$ be two distinct nonconstant meromorphic functions such that $f$ and $g$ share $0,1, \infty C M$, and let $a(\not \equiv 0,1, \infty)$ and $b(\neq 0,1, \infty)$ be two small meromorphic functions of $f$ such that $a \not \equiv b$. If $f-a$ and $g-b$ share $0 C M$, then $f$ is a quasi-Möbius transformation of $g$.

Regarding Theorem $\mathrm{C}$, it is natural to ask the following two questions.

QUeSTION 1.3. What can be said if the condition " $f-a$ and $g-b$ share $0 \mathrm{CM}$ " in Theorem $\mathrm{C}$ is replaced with the condition " $f-a$ and $g-b$ share 0 IM"? 
QUESTION 1.4. What can be said if the condition " $f-a$ and $g-b$ share 0 CM" in Theorem $\mathrm{C}$ is replaced with the condition " $f$ and $g$ share the set $\{a, b\}$ IM"?

In this paper, we will prove the following theorems, which improve Theorems A-C, and deal with Questions 1.1, 1.3 and 1.4.

THEOREM 1.1. Let $f$ and $g$ be two distinct nonconstant meromorphic functions such that $f$ and $g$ share $\left(0, k_{1}\right),\left(1, k_{2}\right)$ and $\left(\infty, k_{3}\right)$, where $k_{1}, k_{2}$ and $k_{3}$ are three positive integers satisfying

$$
k_{1} k_{2} k_{3}>k_{1}+k_{2}+k_{3}+2,
$$

and let $a(\not \equiv 0,1, \infty)$ and $b(\not \equiv 0,1, \infty)$ be two small meromorphic functions of $f$ such that $a \not \equiv b$. Suppose that $f$ and $g$ share the set $\{a, b\} I M$. Then

(I) If $f$ is a quasi-Möbius transformation of $g$, then $f$ and $g$ satisfy one of the following fifteen relations:

(i) $f g=1$ with $a b=1$;

(ix) $f=(1-a) g+a$ with $a b=a+b$;

(ii) $f+g=1$ with $a+b=1$;

(x) $f=\frac{(1-a) g}{1-b}+\frac{b-a}{b-1}$;

(iii) $f=\frac{g}{g-1}$ with $a b=a+b$;

(xi) $f=(1-b) g+b$ with $a b=a+b$;

(iv) $f=\frac{b g}{a}$;

(xii) $f=\frac{a g}{g+a-1}$ with $a+b=1$;

(v) $f=a g$ with $a b=1$;

(xiii) $f=\frac{b(a-1) g}{(a-b) g+(b-1) a}$;

(vi) $f=\frac{a g}{b}$;

(xiv) $f=\frac{b g}{g+b-1}$ with $a+b=1$;

(vii) $f=b g$;

$$
\text { (xv) } f=\frac{a(b-1) g}{(b-a) g+(a-1) b} \text {. }
$$

(viii) $f=\frac{(1-b) g}{1-a}+\frac{a-b}{a-1}$;

(II) If $f$ is not a quasi-Möbius transformation of $g$, then $a$ and $b$ are constants, and there exists a nonconstant entire function $\gamma$ such that $f$ and $g$ are given by one of the following six expressions:

(i) $f=\frac{e^{3 \gamma}-1}{e^{\gamma}-1}, g=\frac{e^{-3 \gamma}-1}{e^{-\gamma}-1}$ with $a=3$ and $b=\frac{3}{4}$, or $a=\frac{3}{4}$ and $b=3$;

(ii) $f=\frac{e^{3 \gamma}-1}{e^{2 \gamma}-1}, g=\frac{e^{-3 \gamma}-1}{e^{-2 \gamma}-1}$ with $a=\frac{3}{2}$ and $b=-3$, or vice versa; 
(iii) $f=\frac{e^{\gamma}-1}{e^{3 \gamma}-1}, g=\frac{e^{-\gamma}-1}{e^{-3 \gamma}-1}$ with $a=\frac{1}{3}$ and $b=\frac{4}{3}$, or vice versa;

(iv) $f=\frac{e^{2 \gamma}-1}{e^{3 \gamma}-1}, g=\frac{e^{-2 \gamma}-1}{e^{-3 \gamma}-1}$ with $a=\frac{2}{3}$ and $b=-\frac{1}{3}$, or vice versa;

(v) $f=\frac{e^{2 \gamma}-1}{e^{-\gamma}-1}, g=\frac{e^{-2 \gamma}-1}{e^{\gamma}-1}$ with $a=-2$ and $b=\frac{1}{4}$, or vice versa;

(vi) $f=\frac{e^{\gamma}-1}{e^{-2 \gamma}-1}, g=\frac{e^{-\gamma}-1}{e^{2 \gamma}-1}$ with $a=-\frac{1}{2}$ and $b=4$, or vice versa.

Using the idea of weighted sharing, we will prove the following theorem which complements Theorem $\mathrm{C}$ and Theorem 1 in [2], and deals with Questions 1.1-1.3.

THEOREM 1.2. Let $f$ and $g$ be two distinct nonconstant entire functions such that $f$ and $g$ share $(0,1)$ and $(1, m)$, where $m(\geq 2)$ is a positive integer, and let $a(\not \equiv 0,1, \infty)$ and $b(\not \equiv 0,1, \infty)$ be two small meromorphic functions of $f$ such that $a \not \equiv b$. If $f=a \Rightarrow g=b$, then $f$ and $g$ satisfy one of the relations $\mathrm{I}(\mathrm{i}), \mathrm{I}(\mathrm{iii}), \mathrm{I}(\mathrm{xii}), \mathrm{I}(\mathrm{xv}), \mathrm{II}(\mathrm{i})$ and $\mathrm{II}(\mathrm{v})$ of Theorem 1.1, where $a=3 / 4$ and $b=3$ in $\mathrm{II}(\mathrm{i})$, and $a=1 / 4$ and $b=-2$ in $\mathrm{II}(\mathrm{v})$.

The following example of Gundersen (see [5]) shows that the condition that $f, g$ share $0,1, \infty \mathrm{CM}$ in Theorem $\mathrm{A}$ cannot be replaced with the condition that $f, g$ share $0,1, \infty$ IM. This example also shows that the condition that $f, g$ share $\left(0, k_{1}\right),\left(1, k_{2}\right)$ and $\left(\infty, k_{3}\right)$ in Theorem 1.1 cannot be replaced with the condition that $f, g$ share $0,1, \infty$ IM.

EXAMPLE 1.1. Let

$$
f(z)=\frac{e^{z}+1}{\left(e^{z}-1\right)^{2}}, \quad g(z)=\frac{\left(e^{z}+1\right)^{2}}{8\left(e^{z}-1\right)} .
$$

Then $f$ and $g$ share $0,1, \infty$ IM. As

$$
f(z)+\frac{1}{2}=\frac{e^{2 z}+3}{2\left(e^{z}-1\right)^{2}}, \quad g(z)-\frac{1}{4}=\frac{e^{2 z}+3}{8\left(e^{z}-1\right)},
$$

we see that $f+1 / 2$ and $g-1 / 4$ share $0 \mathrm{CM}$. However, $f$ is not a bilinear transformation of $g$.

\section{Some lemmas}

Lemma 2.1 (see [13, Lemma 2.6]). Let $f$ and $g$ be two distinct nonconstant meromorphic functions such that $f$ and $g$ share 0,1 and $\infty I M$. If $f$ is quasi-Möbius transformation of $g$, then $f$ and $g$ satisfy one of the 
following relations:

(i) $f \cdot g=1$;

(iv) $f=c g$;

(ii) $(f-1)(g-1)=1$;

(v) $f-1=c(g-1)$;

(iii) $f+g=1$;

(vi) $[(c-1) f+1] \cdot[(c-1) g-c]=-c$;

where $c(\not \equiv 0,1, \infty)$ is a small meromorphic function of $f$.

Let $f$ and $g$ be two distinct nonconstant meromorphic functions, and let $a$ be a value in the extended plane. We denote by $\bar{N}_{0}(r, a)$ the reduced counting function of the common $a$-points of $f$ and $g$. We say that $f$ and $g$ share the value a $I M^{*}$ if

$$
\bar{N}\left(r, \frac{1}{f-a}\right)+\bar{N}\left(r, \frac{1}{g-a}\right)-2 \bar{N}_{0}(r, a)=S(r, f) .
$$

Let $\bar{N}_{E}(r, a)$ "count" those points in $\bar{N}(r, 1 /(f-a))$, where $a$ is taken by $f$ and $g$ with the same multiplicity, and each point is counted only once, and $\bar{N}(r, 1 /(f-\infty))$ means $\bar{N}(r, f)$. We say that $f$ and $g$ share the value a $C M^{*}$ if

$$
\bar{N}\left(r, \frac{1}{f-a}\right)+\bar{N}\left(r, \frac{1}{g-a}\right)-2 \bar{N}_{E}(r, a)=S(r, f) .
$$

If $a(\not \equiv 0,1, \infty)$ is a small meromorphic function of $f$ and $g$, the above definitions are still valid. Let $f$ and $g$ share 0,1 and $\infty$ IM. We denote by $N_{0}(r)$ the counting function of the zeros of $f-g$ not containing the zeros of $f, 1 / f$ and $f-1$.

Lemma 2.2 (see [16, Theorem 1.1]). Let $f$ and $g$ be two nonconstant meromorphic functions, and let $a_{1}, a_{2}, a_{3}, a_{4}$ and $a_{5}$ be five distinct elements in the set $\{S(f) \cap S(g)\} \cup\{\infty\}$, where $S(f)$ is the set of meromorphic functions which are small functions of $f$. If $f$ and $g$ share $a_{1}, a_{2}, a_{3}, a_{4}$ and $a_{5} I M^{*}$, then $f=g$.

Lemma 2.3 (see [17, Lemma 2.6]). Let $f$ and $g$ be two distinct nonconstant meromorphic functions sharing $\left(0, k_{1}\right),\left(1, k_{2}\right)$ and $\left(\infty, k_{3}\right)$, where $k_{1}$, $k_{2}$ and $k_{3}$ are three positive integers satisfying (1.1). Then

$$
\bar{N}_{(2}\left(r, \frac{1}{f}\right)+\bar{N}_{(2}\left(r, \frac{1}{f-1}\right)+\bar{N}_{(2}(r, f)=S(r, f) .
$$

Lemma 2.4 (see [18, Lemma 6]). Let $f_{1}$ and $f_{2}$ be nonconstant meromorphic functions satisfying $\bar{N}\left(r, f_{j}\right)+\bar{N}\left(r, 1 / f_{j}\right)=S(r)(j=1,2)$. Then either $\bar{N}_{0}\left(r, 1 ; f_{1}, f_{2}\right)=S(r)$ or there exist two integers $s, t(|s|+|t|>0)$ such that $f_{1}^{s} f_{2}^{t}=1$. Here and below, $\bar{N}_{0}\left(r, 1 ; f_{1}, f_{2}\right)$ denotes the reduced counting function of $f_{1}$ and $f_{2}$ related to the common 1-points and $T(r)=$ $T\left(r, f_{1}\right)+T\left(r, f_{2}\right), S(r)=o(T(r))(r \rightarrow \infty, r \notin E)$ only depending on $f_{1}$ and $f_{2}$. 
Lemma 2.5 (see [18, proof of Theorems 1 and 2]). Let $f$ and $g$ be two distinct nonconstant meromorphic functions sharing 0,1 and $\infty C M$, and let $N_{0}(r) \neq S(r, f)$. If $f$ is a fractional linear transformation of $g$, then $N_{0}(r)=T(r, f)+S(r, f)$. If $f$ is not a fractional linear transformation of $g$, then $N_{0}(r) \leq \frac{1}{2} T(r, f)+S(r, f)$, and $f$ and $g$ satisfy one of the following three relations:
(i) $f=\frac{e^{(k+1) \gamma}-1}{e^{s \gamma}-1}$,
$g=\frac{e^{-(k+1) \gamma}-1}{e^{-s \gamma}-1}$,
(ii) $f=\frac{e^{s \gamma}-1}{e^{(k+1) \gamma}-1}$,

$$
g=\frac{e^{-s \gamma}-1}{e^{-(k+1) \gamma}-1}
$$
(iii) $f=\frac{e^{s \gamma}-1}{e^{-(k+1-s) \gamma}-1}$,

$$
g=\frac{e^{-s \gamma}-1}{e^{(k+1-s) \gamma}-1}
$$

where $\gamma$ is a nonconstant entire function, $s$ and $k(\geq 2)$ are positive integers such that $s$ and $k+1$ are relatively prime and $1 \leq s \leq k$.

Lemma 2.6 (see [18]). Let $s(>0)$ and $t$ be relatively prime integers, and let $c$ be a finite complex number such that $c^{s}=1$. Then there exists one and only one common zero of $\omega^{s}-1$ and $\omega^{t}-c$.

Lemma 2.7 (see [14]). Let $f$ be a nonconstant meromorphic function, and let $F=\sum_{k=0}^{p} a_{k} f^{k} / \sum_{j=0}^{q} b_{j} f^{j}$ be an irreducible rational function in $f$ with constant coefficients $\left\{a_{k}\right\}$ and $\left\{b_{j}\right\}$, where $a_{p} \neq 0$ and $b_{q} \neq 0$. Then $T(r, F)=d T(r, f)+O(1)$, where $d=\max \{p, q\}$.

Lemma 2.8 (see [15, Theorem 1.62]). Let $f_{1}, \ldots, f_{n}$ be nonconstant meromorphic functions, and let $f_{n+1}(\not \equiv 0)$ be a meromorphic function such that $\sum_{i=1}^{n+1} f_{i}=1$. If there exists a subset $I \subseteq \mathbb{R}^{+}$satisfying mes $I=\infty$ such that

$$
\begin{aligned}
\sum_{i=1}^{n+1} N\left(r, 1 / f_{i}\right)+n & \sum_{\substack{i=1 \\
i \neq j}}^{n+1} \bar{N}\left(r, f_{i}\right) \\
& <(\lambda+o(1)) T\left(r, f_{j}\right) \quad(r \rightarrow \infty, r \in I, 1 \leq j \leq n),
\end{aligned}
$$

where $\lambda<1$, then $f_{n+1}=1$.

Lemma 2.9. Let $f$ and $g$ be two distinct nonconstant meromorphic functions such that $f$ and $g$ share $\left(0, k_{1}\right),\left(1, k_{2}\right)$ and $\left(\infty, k_{3}\right)$, where $k_{1}, k_{2}$ and $k_{3}$ are three positive integers satisfying $(1.1)$, and let $a(\not \equiv 0,1, \infty)$ be a small meromorphic function of $f$. Then either

$$
N_{(3}(r, 1 /(f-a))+N_{(3}(r, 1 /(g-a))=S(r, f),
$$


or $f$ and $g$ are given by one of the following six expressions:

(i) $g=a f ; \quad$ (iv) $f+(a-1) g=a$;

(ii) $g+(a-1) f=a ; \quad(\mathrm{v})(g-a)(f+a-1)=a(1-a)$;

(iii) $f=a g$;

(vi) $(f-a)(g+a-1)=a(1-a)$.

Proof. First, from the condition that $f$ and $g$ share $0,1, \infty$ IM, we have $T(r, f) \leq 3 T(r, g)+S(r, f)$ and $T(r, g) \leq 3 T(r, f)+S(r, g)$, and so

$$
S(r, g)=S(r, f) \text {. }
$$

Let

$$
(f-1) /(g-1)=\alpha \quad \text { and } \quad f / g=h .
$$

Then from (2.2), (2.3) and Lemma 2.3 we get

$$
\bar{N}(r, 1 / \alpha)+\bar{N}(r, \alpha)=S(r, f) \quad \text { and } \quad \bar{N}(r, 1 / h)+\bar{N}(r, h)=S(r, f) .
$$

If one of $\alpha=h, \alpha=1$ and $h=1$ holds, from (2.3) we get $f=g$, which contradicts the assumption of Lemma 2.9. Next we suppose that $\alpha \neq \equiv h$, $\alpha \neq \equiv 1$ and $h \not \equiv 1$. Applying (2.3) we deduce

$$
f=\frac{1-\alpha^{-1}}{h^{-1}-\alpha^{-1}} \quad \text { and } \quad g=\frac{1-\alpha}{h-\alpha} .
$$

We discuss the following five cases.

CASE 1. Suppose that $a^{\prime} h+a h^{\prime}=0$. Then $(a h)^{\prime}=0$, and so $a h=A_{1}$, where $A_{1}(\neq 0)$ is a finite complex number. Applying (2.3), we have

$$
f / g=A_{1} / a \text { and } T(r, h)=S(r, f) .
$$

Since

$$
h-1=(f-g) / g,
$$

from (2.2), (2.6), (2.7) and the condition that $f$ and $g$ share 1 IM, we have

$$
\begin{aligned}
\bar{N}(r, 1 /(f-1)) & =\bar{N}(r, 1 /(g-1)) \leq \bar{N}(r, 1 /(h-1)) \\
& \leq T(r, h)+O(1)=S(r, f) .
\end{aligned}
$$

From (2.6) and $f \not \equiv g$, and the condition that $f$ and $g$ share 1 IM, we get

$$
A_{1} / a \not \equiv 1 \text { and } \bar{N}\left(r, 1 /\left(g-a / A_{1}\right)\right)=S(r, f) \text {. }
$$

If $a / A_{1}=a$, then $A_{1}=1$, and so from (2.6) we have (i) of Lemma 2.9. If $a / A_{1} \not \equiv a$, from (2.2), (2.8), (2.9) and Nevanlinna's three small functions theorem (see [15, Theorem 1.36]), we get

$$
T(r, g)=\bar{N}(r, 1 /(g-a))+S(r, f)=N(r, 1 /(g-a))+S(r, f) .
$$

From $(2.10)$ we get $N_{(2}(r, 1 /(g-a))=S(r, f)$, and so

$$
N_{(3}(r, 1 /(g-a))=S(r, f) .
$$


CASE 2. Suppose that $(a-1) \alpha^{\prime}+a^{\prime} \alpha=0$. Then $((a-1) \alpha)^{\prime}=0$, and so $(a-1) \alpha=A_{2}$, where $A_{2}(\neq 0)$ is a finite complex number. From this and (2.3) we have

$$
(f-1) /(g-1)=A_{2} /(a-1) .
$$

From (2.12), the condition $f \not \equiv g$ and the condition that $f$ and $g$ share 0 IM, we get

$$
A_{2} /(a-1) \not \equiv 1
$$

and

$$
\begin{aligned}
\bar{N}(r, 1 / f) & =\bar{N}(r, 1 / g) \leq \bar{N}\left(r, \frac{1}{A_{2} /(a-1)-1}\right) \\
& \leq T(r, a)+O(1)=S(r, f) .
\end{aligned}
$$

Since (2.12) can be rewritten as

$$
f=\frac{A_{2}}{a-1} \cdot\left(g-\frac{A_{2}-(a-1)}{A_{2}}\right),
$$

from $(2.13),(2.15)$ and the condition that $f$ and $g$ share 0 IM, we get

$$
\frac{A_{2}-(a-1)}{A_{2}} \not \equiv 0 \text { and } \bar{N}\left(r, 1 /\left(g-\frac{A_{2}-(a-1)}{A_{2}}\right)\right)=S(r, f) \text {. }
$$

If $\left(A_{2}-(a-1)\right) / A_{2}=a$, then $A_{2}=-1$. From this and (2.12) we have (ii) of Lemma 2.9. If $\left(A_{2}-(a-1)\right) / A_{2} \not \equiv a$, from (2.14), (2.16) and Nevanlinna's three small functions theorem, we get (2.10) and (2.11).

CASE 3. Suppose that $a^{\prime} h^{-1}+a\left(h^{-1}\right)^{\prime}=0$. Proceeding as in Case 1, we get (iii) of Lemma 2.9.

Case 4. Suppose that $(a-1)\left(\alpha^{-1}\right)^{\prime}+a^{\prime} \alpha^{-1}=0$. Proceeding as in Case 2, we get (iv) of Lemma 2.9.

Case 5. Suppose that

$$
\begin{aligned}
& a^{\prime} h+a h^{\prime} \not \equiv 0, \quad(a-1) \alpha^{\prime}+a^{\prime} \alpha \not \equiv 0, \\
& a^{\prime} h^{-1}+a\left(h^{-1}\right)^{\prime} \not \equiv 0, \quad(a-1)\left(\alpha^{-1}\right)^{\prime}+a^{\prime} \alpha^{-1} \not \equiv 0 .
\end{aligned}
$$

From (2.5) we get

$$
g-a=\frac{1-a h+(a-1) \alpha}{h-\alpha} .
$$

Let

$$
\omega=1-a h+(a-1) \alpha .
$$

By differentiating both sides of (2.20) twice, we get

$$
\omega^{\prime}=\left\{(a-1) \cdot \frac{\alpha^{\prime}}{\alpha}+a^{\prime}\right\} \cdot \alpha-\left(a^{\prime}+a \cdot \frac{h^{\prime}}{h}\right) \cdot h,
$$




$$
\omega^{\prime \prime}=\left\{2 a^{\prime} \cdot \frac{\alpha^{\prime}}{\alpha}+(a-1) \cdot \frac{\alpha^{\prime \prime}}{\alpha}+a^{\prime \prime}\right\} \cdot \alpha-\left(a^{\prime \prime}+2 a^{\prime} \cdot \frac{h^{\prime}}{h}+a \cdot \frac{h^{\prime \prime}}{h}\right) \cdot h .
$$

We discuss the following three subcases.

Subcase 5.1. Suppose that $D=0$, where

$$
D=\left|\begin{array}{cc}
-a^{\prime}-a \cdot \frac{h^{\prime}}{h} & (a-1) \cdot \frac{\alpha^{\prime}}{\alpha}+a^{\prime} \\
-a^{\prime \prime}-2 a^{\prime} \cdot \frac{h^{\prime}}{h}-a \cdot \frac{h^{\prime \prime}}{h} & 2 a^{\prime} \cdot \frac{\alpha^{\prime}}{\alpha}+(a-1) \cdot \frac{\alpha^{\prime \prime}}{\alpha}+a^{\prime \prime}
\end{array}\right| .
$$

Then from $(2.23)$ we get

$$
\left(a^{\prime} h+a h^{\prime}\right) \cdot\left((a-1) \cdot \alpha^{\prime}+a^{\prime} \cdot \alpha\right)^{\prime}=\left((a-1) \cdot \alpha^{\prime}+a^{\prime} \cdot \alpha\right) \cdot\left(a^{\prime} h+a h^{\prime}\right)^{\prime} .
$$

From (2.17) and (2.24) we get

$$
\frac{\left((a-1) \alpha^{\prime}+a^{\prime} \alpha\right)^{\prime}}{(a-1) \alpha^{\prime}+a^{\prime} \alpha}=\frac{\left(a^{\prime} h+a h^{\prime}\right)^{\prime}}{a^{\prime} h+a h^{\prime}} .
$$

From (2.25) we get

$$
(a-1) \alpha^{\prime}+a^{\prime} \alpha=A_{3} \cdot\left(a^{\prime} h+a h^{\prime}\right),
$$

where $A_{3}(\neq 0)$ is a finite complex number. From (2.26) we deduce

$$
(a-1) \cdot \alpha=A_{3} a h+A_{4},
$$

where $A_{4}$ is a finite complex number. If there exists a subset $I \subseteq \mathbb{R}^{+}$satisfying mes $I=\infty$ such that $T(r, \alpha)=S(r, f)(r \in I, r \rightarrow \infty)$, then it follows by (2.27) that $T(r, h)=S(r, f)(r \in I, r \rightarrow \infty)$, and so from (2.5) we have $T(r, f)=S(r, f)(r \in I, r \rightarrow \infty)$, which is impossible. Thus from (2.27) we have

$$
T(r, \alpha) \neq S(r, f) \quad \text { and } \quad T(r, h) \neq S(r, f) \quad(r \notin E, r \rightarrow \infty) .
$$

Next we put $\gamma_{0}:=\alpha / h$. If $A_{4} \neq 0$, from (2.4), (2.27), (2.28) and Lemma 2.8 we get a contradiction. Thus $A_{4}=0$. Applying (2.3) and (2.27) we have

$$
\frac{f-1}{f}=\gamma_{0} \cdot \frac{g-1}{g}
$$

where $\gamma_{0}=\left(A_{3} a\right) /(a-1)$. If $\bar{N}(r, f) \neq S(r, f)$, from (2.29) and the condition that $f$ and $g$ share $\infty \mathrm{IM}$, we get $\left(A_{3} a\right) /(a-1)=1$, and so it follows by $(2.29)$ that $f=g$, which is a contradiction. Thus

$$
\bar{N}(r, f)=\bar{N}(r, g)=S(r, f) .
$$

Since (2.29) can be rewritten as

$$
\frac{f}{g} \cdot\left(g-\frac{\gamma_{0}}{\gamma_{0}-1}\right)=\frac{1}{1-\gamma_{0}},
$$

from (2.31) and Lemma 2.3 we get

$$
\bar{N}\left(r, \frac{1}{g-\gamma_{0} /\left(\gamma_{0}-1\right)}\right)=S(r, f) .
$$


If $\gamma_{0} /\left(\gamma_{0}-1\right) \not \equiv a$, from (2.30), (2.32) and Nevanlinna's three small functions theorem we get (2.10) and (2.11). If $\gamma_{0} /\left(\gamma_{0}-1\right)=a$, then $\gamma_{0}=a /(a-1)$. From this and (2.29) we get (v) of Lemma 2.9.

SuBCASE 5.2. Suppose that $D_{1}=0$, where

$$
D_{1}=\left|\begin{array}{cc}
-a^{\prime}-a \cdot \frac{h_{1}^{\prime}}{h_{1}} & (a-1) \cdot \frac{\alpha_{1}^{\prime}}{\alpha_{1}}+a^{\prime} \\
-a^{\prime \prime}-2 a^{\prime} \cdot \frac{h_{1}^{\prime}}{h_{1}}-a \cdot \frac{h_{1}^{\prime \prime}}{h_{1}} & 2 a^{\prime} \cdot \frac{\alpha_{1}^{\prime}}{\alpha_{1}}+(a-1) \cdot \frac{\alpha_{1}^{\prime \prime}}{\alpha_{1}}+a^{\prime \prime}
\end{array}\right|,
$$

and $h_{1}=h^{-1}, \alpha_{1}=\alpha^{-1}$. From $D_{1}=0$, in the same manner as in Subcase 5.1, we get (vi) of Lemma 2.9.

Subcase 5.3. Suppose that $D \not \equiv 0$ and $D_{1} \not \equiv 0$, where $D$ is defined by (2.23), and $D_{1}$ is defined by (2.33). First, we will prove (2.11). Let

$$
\omega=1-a h+(a-1) \alpha .
$$

By differentiating both sides of (2.34) two times we get

$$
\begin{aligned}
\omega^{\prime} & =\left\{(a-1) \cdot \frac{\alpha^{\prime}}{\alpha}+a^{\prime}\right\} \cdot \alpha-\left(a^{\prime}+a \cdot \frac{h^{\prime}}{h}\right) \cdot h, \\
\omega^{\prime \prime} & =\left\{2 a^{\prime} \cdot \frac{\alpha^{\prime}}{\alpha}+(a-1) \cdot \frac{\alpha^{\prime \prime}}{\alpha}+a^{\prime \prime}\right\} \cdot \alpha-\left(a^{\prime \prime}+2 a^{\prime} \cdot \frac{h^{\prime}}{h}+a \cdot \frac{h^{\prime \prime}}{h}\right) \cdot h .
\end{aligned}
$$

From (2.35) and (2.36) we get

$$
\alpha=\frac{D_{\alpha}}{D} \quad \text { and } \quad h=\frac{D_{h}}{D},
$$

where

$$
\begin{aligned}
& D_{\alpha}=\left|\begin{array}{cc}
-a^{\prime}-a \cdot \frac{h^{\prime}}{h} & \omega^{\prime} \\
-a^{\prime \prime}-2 a^{\prime} \cdot \frac{h^{\prime}}{h}-a \cdot \frac{h^{\prime \prime}}{h} & \omega^{\prime \prime}
\end{array}\right|, \\
& D_{h}=\left|\begin{array}{cc}
\omega^{\prime} & (a-1) \cdot \frac{\alpha^{\prime}}{\alpha}+a^{\prime} \\
\omega^{\prime \prime} & 2 a^{\prime} \cdot \frac{\alpha^{\prime}}{\alpha}+(a-1) \cdot \frac{\alpha^{\prime \prime}}{\alpha}+a^{\prime \prime}
\end{array}\right| .
\end{aligned}
$$

Substituting (2.37) into (2.34) we get

$$
\omega+y_{1} \cdot \omega^{\prime}+y_{2} \cdot \omega^{\prime \prime}=1,
$$

where

$$
\begin{aligned}
& y_{1}=\frac{a(a-1) \cdot \frac{\alpha^{\prime \prime}}{\alpha}+a a^{\prime \prime}+2 a a^{\prime} \cdot \frac{\alpha^{\prime}}{\alpha}+(1-a) \cdot\left(a^{\prime \prime}+2 a^{\prime} \cdot \frac{h^{\prime}}{h}+a \cdot \frac{h^{\prime \prime}}{h}\right)}{D} \\
& y_{2}=\frac{(a-1)\left(a^{\prime}+a \cdot \frac{h^{\prime}}{h}\right)-a(a-1) \cdot \frac{\alpha^{\prime}}{\alpha}-a a^{\prime}}{D}
\end{aligned}
$$


From $(2.2),(2.4),(2.23),(2.39),(2.40)$ and the lemma of logarithmic derivative (see [10, Corollary 2.3.4]), we get

$$
T\left(r, y_{1}\right)+T\left(r, y_{2}\right)=S(r, f) .
$$

On the other hand, from (2.4) we get

$$
\begin{aligned}
\bar{N}(r, \alpha / h)+\bar{N}(r, h / \alpha) & \leq \bar{N}(r, \alpha)+\bar{N}(r, 1 / h)+\bar{N}(r, h)+\bar{N}(r, 1 / \alpha) \\
& =S(r, f) .
\end{aligned}
$$

Noting that $h / \alpha \not \equiv 1$, from (2.42) and the second fundamental theorem, we get

$$
T(r, h / \alpha)=\bar{N}\left(r, \frac{1}{h / \alpha-1}\right)+S(r, f)=N\left(r, \frac{1}{h / \alpha-1}\right)+S(r, f) .
$$

From (2.43) we deduce

$$
N_{(2}\left(r, \frac{1}{h / \alpha-1}\right)=S(r, f) .
$$

From (2.19), (2.34), (2.38), (2.41), (2.44) and the left equality of (2.4), we get (2.11). Similarly, from $D_{1} \not \equiv 0$ we get

$$
N_{(3}(r, 1 /(f-a))=S(r, f) .
$$

From (2.11) and (2.45) we have (2.1).

Lemma 2.9 is thus completely proved.

Lemma 2.10 (see [11, Theorem 3]). Let $f$ and $g$ be two distinct nonconstant meromorphic functions such that $f$ and $g$ share 0,1 and $\infty C M^{*}$, and let $a(\not \equiv 0,1, \infty)$ be a small meromorphic function. If $T(r, f) \neq$ $N(r, 1 /(f-a))+S(r, f)$, then one of the following cases will occur:

(i) $f=a g$ and $\bar{N}(1,1 /(f-a))+\bar{N}(1,1 /(f-1))=S(r, f)$;

(ii) $f-1=(1-a)(g-1)$ and $\bar{N}(1,1 /(f-a))+\bar{N}(1,1 / f)=S(r, f)$;

(iii) $(f-a)(g-1+a)=a(1-a)$ and $\bar{N}(r, 1 /(f-a))+\bar{N}(r, f)=S(r, f)$.

Let $f$ and $g$ be two distinct nonconstant meromorphic functions, and let $a(\not \equiv 0,1, \infty)$ and $b(\not \equiv 0,1, \infty)$ be small meromorphic functions such that $a \not \equiv b$. We denote by $\bar{N}_{0}(r, a, b)$ the reduced counting function of the common zeros of $f-a$ and $g-b$, and by $\bar{N}_{(l, k)}(r, a, b)$ the reduced counting function of those zeros of $f-a$ with multiplicity $l$, and of $g-b$ with multiplicity $k$.

Lemma 2.11 (see [12, Theorem 4.2]). Let $f$ and $g$ be two distinct nonconstant meromorphic functions sharing 0,1 and $\infty$ CM. If there exists a finite complex number $a(\neq 0,1)$ such that $a$ is not a Picard value of $f$, and such that $N_{1)}(r, 1 /(f-a)) \leq u T(r, f)+S(r, f)$, where $u<1 / 3$, then $N_{1)}(r, 1 /(f-a))=0$, and $f, g$ are given by one of the following nine ex- 
pressions:

(i) $f=\frac{e^{3 \gamma}-1}{e^{\gamma}-1}, g=\frac{e^{-3 \gamma}-1}{e^{-\gamma}-1}$ with $a=\frac{3}{4}$;

(ii) $f=\frac{e^{3 \gamma}-1}{e^{2 \gamma}-1}, g=\frac{e^{-3 \gamma}-1}{e^{-2 \gamma}-1}$ with $a=-3$;

(iii) $f=\frac{e^{\gamma}-1}{e^{3 \gamma}-1}, g=\frac{e^{-\gamma}-1}{e^{-3 \gamma}-1}$ with $a=\frac{4}{3}$;

(iv) $f=\frac{e^{2 \gamma}-1}{e^{3 \gamma}-1}, g=\frac{e^{-2 \gamma}-1}{e^{-3 \gamma}-1}$ with $a=-\frac{1}{3}$;

(v) $f=\frac{e^{2 \gamma}-1}{e^{-\gamma}-1}, g=\frac{e^{-2 \gamma}-1}{e^{\gamma}-1}$ with $a=\frac{1}{4}$;

(vi) $f=\frac{e^{\gamma}-1}{e^{-2 \gamma}-1}, g=\frac{e^{-\gamma}-1}{e^{2 \gamma}-1}$ with $a=4$;

(vii) $f=\frac{e^{2 \gamma}-1}{\lambda e^{\gamma}-1}, g=\frac{e^{-2 \gamma}-1}{\frac{1}{\lambda} e^{-\gamma}-1}$ with $\lambda^{2} \neq 1$ and $a^{2} \lambda^{2}=4(a-1)$;

(viii) $f=\frac{e^{\gamma}-1}{\lambda e^{2 \gamma}-1}, g=\frac{e^{-\gamma}-1}{\frac{1}{\lambda} e^{-2 \gamma}-1}$ with $\lambda \neq 1$ and $4 a(1-a) \lambda=1$;

(ix) $f=\frac{e^{\gamma}-1}{\lambda e^{-\gamma}-1}, g=\frac{e^{-\gamma}-1}{\frac{1}{\lambda} e^{\gamma}-1}$ with $\lambda \neq 1$ and $(1-a)^{2}+4 a \lambda=0$; where $\gamma$ is a nonconstant entire function.

From Lemmas 2.3 and 2.9 we get the following result.

LEMMA 2.12 (see [11, proof of Theorem 6]). Let $f$ and $g$ be two distinct nonconstant meromorphic functions such that $f$ and $g$ share $\left(0, k_{1}\right),\left(1, k_{2}\right)$ and $\left(\infty, k_{3}\right)$, where $k_{1}, k_{2}$ and $k_{3}$ are three positive integers satisfying (1.1), and let $a(\not \equiv 0,1, \infty)$ and $b(\not \equiv 0,1, \infty)$ be two small meromorphic functions of $f$. If

$$
\bar{N}_{(2,1)}(r, a, b)+\bar{N}_{(1,2)}(r, a, b)=S(r, f)
$$

and

$$
\bar{N}(r, 1 /(f-a))+\bar{N}(r, 1 /(g-b))-2 \bar{N}_{0}(r, a, b)=S(r, f),
$$

then $f$ is a quasi-Möbius transformation of $g$.

\section{Proofs}

Proof of Theorem 1.1. We discuss the following two cases.

CAsE 1. Suppose that $f$ is a quasi-Möbius transformation of $g$. Then $f$ and $g$ satisfy one of the six relations (i)-(vi) in Lemma 2.1. We discuss the following two subcases. 
SubCASE 1.1. Suppose that

$$
\bar{N}_{0}(r, a) \neq S(r, f) .
$$

Then from (3.1) and the six relations (i)-(vi) in Lemma 2.1 we see that $f-a$ and $g-a$ share $0 \mathrm{CM}^{*}$. From this and the condition that $f$ and $g$ share the set $\{a, b\}$ IM, we see that $f-b$ and $g-b$ also share $0 \mathrm{CM}^{*}$. Noting that $f$ and $g$ share 0,1 and $\infty$ IM, by Lemma 2.2 we get $f=g$, which contradicts the assumptions of Theorem 1.1.

SubCASE 1.2. Suppose that

$$
\bar{N}_{0}(r, b) \neq S(r, f) .
$$

Then in the same manner as in Subcase 1.1 we get a contradiction.

SubCASE 1.3. Suppose that

$$
\bar{N}_{0}(r, a)+\bar{N}_{0}(r, b)=S(r, f) .
$$

Noting that $f$ and $g$ share the set $\{a, b\}$ IM, from (3.3) and the six relations (i)-(vi) in Lemma 2.1 we see that $f-a$ and $g-b$ share $0 \mathrm{CM}^{*}$, and that $f-b$ and $g-a$ share $0 \mathrm{CM}^{*}$. We discuss the following four subcases.

SubCASE 1.3.1. Suppose that $f$ and $g$ satisfy one of the three relations (i)-(iii) in Lemma 2.1. Then two of 0,1 and $\infty$ are exceptional small functions of $f$. From this and the condition that $f-a$ and $g-b$ share 0 $\mathrm{CM}^{*}$, and the condition that $f-b$ and $g-a$ share $0 \mathrm{CM}^{*}$, we see that $\bar{N}(r, 1 /(f-a))=\bar{N}(r, 1 /(g-b))+S(r, f) \neq S(r, f)$ and $\bar{N}(r, 1 /(f-b))=$ $\bar{N}(r, 1 /(g-a))+S(r, f) \neq S(r, f)$. From this we get I(i)-(iii) of Theorem 1.1 respectively.

SubCASE 1.3.2. Suppose that $f$ and $g$ satisfy the relation (iv) of Lemma 2.1. Then it follows that $1, c$ are two exceptional small functions of $f$, and $1,1 / c$ are two exceptional small functions of $g$. If $\bar{N}(r, 1 /(f-a))+$ $\bar{N}(r, 1 /(g-b))=S(r, f)$, then $\bar{N}(r, 1 /(f-b))=\bar{N}(r, 1 /(g-a))+S(r, f) \neq$ $S(r, f)$, and so $a=c$ and $b=1 / c$. From this we get $\mathrm{I}(\mathrm{iv})$ and $\mathrm{I}(\mathrm{v})$ of Theorem 1.1. Similarly, if $\bar{N}(r, 1 /(f-a))=\bar{N}(r, 1 /(g-b))+S(r, f) \neq S(r, f)$ and $\bar{N}(r, 1 /(f-b))+\bar{N}(r, 1 /(g-a))=S(r, f)$, then $b=c$ and $a=1 / c$, and so we have I(vi) and I(vii) of Theorem 1.1. If $\bar{N}(r, 1 /(f-a))=\bar{N}(r, 1 /(g-b))+$ $S(r, f) \neq S(r, f)$ and $\bar{N}(r, 1 /(f-b))=\bar{N}(r, 1 /(g-a))+S(r, f) \neq S(r, f)$, then we have I(iv) and I(vi) of Theorem 1.1.

SubCASE 1.3.3. Suppose that $f$ and $g$ satisfy the relation (v) of Lemma 2.1. Then it follows that 0 is the only exceptional small function of $f$ and $g$, and $f, g$ are given by

$$
f=\frac{c-1}{e^{\gamma}-1}, \quad g=\frac{c^{-1}-1}{e^{-\gamma}-1},
$$


where $\gamma$ is a nonconstant entire function. From (3.4) we deduce

$$
f-a=\frac{-a e^{\gamma}+(a+c-1)}{e^{\gamma}-1}, \quad g-b=\frac{-b e^{-\gamma}+\left(b+c^{-1}-1\right)}{e^{-\gamma}-1} .
$$

From (3.5) and the relation (v) of Lemma 2.1, in the same manner as in Subcase 1.3.2 we get the conclusions I(viii)-(xi) of Theorem 1.1.

SubCASE 1.3.4. Suppose that $f$ and $g$ satisfy the relation (vi) of Lemma 2.1. Then it follows that $\infty$ is the only exceptional small function of $f$ and $g$, and $f$ and $g$ are given by

$$
f=\frac{e^{\gamma}-1}{c-1}, \quad g=\frac{e^{-\gamma}-1}{c^{-1}-1},
$$

where $\gamma$ is a nonconstant entire function. From (3.6) we deduce

$$
f-a=\frac{e^{\gamma}-(1+a(c-1))}{c-1}, \quad g-b=\frac{e^{-\gamma}-\left(1+b\left(c^{-1}-1\right)\right)}{c^{-1}-1} .
$$

From (3.7) and the relation (vi) of Lemma 2.1, in the same manner as in Subcase 1.3.2 we get the conclusions I(xii)-(xv) of Theorem 1.1.

CASE 2. Suppose that $f$ is not a quasi-Möbius transformation of $g$. From the condition that $f$ and $g$ share 0,1 and $\infty$ IM we get

$$
S(r, f)=S(r, g) \text {. }
$$

Let

$$
\begin{aligned}
\frac{f-1}{g-1} & =h_{1}, \\
\frac{f}{g} & =h_{2}, \\
h_{0} & =\frac{h_{1}}{h_{2}} .
\end{aligned}
$$

From (3.8)-(3.11) and Lemma 2.3 we get

$$
\bar{N}\left(r, h_{j}\right)+\bar{N}\left(r, 1 / h_{j}\right)=S(r, f) \quad(j=0,1,2) .
$$

Noting that $f$ is not a Möbius transformation of $g$, from (3.8)-(3.11) we see that none of $h_{1}, h_{2}$ and $h_{0}$ is constant. From (3.9)-(3.11) we get

$$
\begin{aligned}
& f=\frac{h_{1}-1}{h_{0}-1}, \\
& g=\frac{h_{1}^{-1}-1}{h_{0}^{-1}-1} .
\end{aligned}
$$

From (3.9), (3.10), (3.13) and (3.14) we get

$$
f-g=\frac{\left(h_{1}-1\right)\left(1-h_{0} h_{1}^{-1}\right)}{h_{0}-1} .
$$


From (3.8) and (3.11)-(3.15) we get

$$
N_{0}(r)=N_{0}\left(r, 1 ; h_{1}, h_{0}\right)+S(r, f)=N_{0}\left(r, 1 ; h_{1}, h_{2}\right)+S(r, f) .
$$

We discuss the following three subcases.

SubCaSe 2.1. Suppose that (3.1) and

$$
\bar{N}_{0}(r, b)=S(r, f) \text {. }
$$

Then from (3.1) we get

$$
N_{0}(r) \neq S(r, f) .
$$

From (3.16) and (3.18) we get

$$
N_{0}\left(r, 1 ; h_{1}, h_{2}\right) \neq S(r, f) .
$$

From (3.12), (3.19) and Lemma 2.4 we see that there exist two integers $s$ and $t(|s|+|t|>0)$ such that

$$
h_{1}^{s} h_{2}^{t}=1 \text {. }
$$

Substituting (3.9) and (3.10) into (3.20) we get

$$
f^{t}(f-1)^{s}=g^{t}(g-1)^{s} .
$$

Noting that $f$ is not a Möbius transformation of $g$, from (3.21) we deduce that $s \neq 0$, and $t \neq 0$ and $|s| \neq|t|$, and so it follows from (3.21) that $f$ and $g$ share 0,1 and $\infty$ CM. Noting that $f$ is not a Möbius transformation of $g$, from (3.18) and Lemma 2.5 we see that $f$ and $g$ are given by one of the three expressions (i)-(iii) of Lemma 2.5. Applying (3.17), Lemmas 2.6, 2.7, $2.9,2.10$, and the condition that $f$ and $g$ share the set $\{a, b\}$ IM, we get

$$
\begin{aligned}
& T(r, f)-T(r, g)=N_{2)}(r, 1 /(f-a))-N_{2)}(r, 1 /(g-b))=S(r, f), \\
& T(r, g)-T(r, f)=N_{2)}(r, 1 /(g-a))-N_{2)}(r, 1 /(f-b))=S(r, f) .
\end{aligned}
$$

Let

$$
\varphi=\frac{f^{\prime}(f-a)}{f(f-1)}-\frac{g^{\prime}(g-a)}{g(g-1)} .
$$

Noting that $f$ and $g$ share 0,1 and $\infty \mathrm{CM}$, from (3.8) and (3.24) we get $T(r, \varphi)=S(r, f)$. Applying (3.1) and (3.24) we get $\varphi=0$, which reads

$$
\frac{f^{\prime}(f-a)}{f(f-1)}=\frac{g^{\prime}(g-a)}{g(g-1)},
$$

and (3.25) can be rewritten as

$$
\frac{f^{\prime}}{f-1}-\frac{g^{\prime}}{g-1}=\frac{a}{a-1} \cdot\left(\frac{f^{\prime}}{f}-\frac{g^{\prime}}{g}\right)
$$

From (3.26) and (i)-(iii) of Lemma 2.5 we see that $a$ is a constant. Let $z_{0}$ be a zero of $g-a$ with multiplicity 2 , and a zero of $f-b$ with multiplicity $\leq 2$. 
Then it follows from (3.23) and (3.25) that $f^{\prime}\left(z_{0}\right)=g^{\prime}\left(z_{0}\right)=0$. Applying (3.8), (3.9), (3.12) and Lemma 2.9, we get

$$
\begin{aligned}
N_{(2}(r, & 1 /(g-a)) \\
& =\left\{N_{(2}\left(r, \frac{1}{g-a}\right)-N_{(3}\left(r, \frac{1}{g-a}\right)\right\}+N_{(3}\left(r, \frac{1}{g-a}\right) \\
& \leq 2 \bar{N}\left(r, \frac{h_{1}}{h_{1}^{\prime}}\right)+S(r, f) \leq 2 T\left(r, \frac{h_{1}^{\prime}}{h_{1}}\right)+S(r, f) \\
& =2\left\{m\left(r, \frac{h_{1}^{\prime}}{h_{1}}\right)+N\left(r, \frac{h_{1}^{\prime}}{h_{1}}\right)\right\}+S(r, f)=S(r, f) .
\end{aligned}
$$

Similarly, from (3.22) and (3.25) we get

$$
N_{(2}(r, 1 /(f-a))=S(r, f) .
$$

If $b$ is not a constant, then $b^{\prime} \not \equiv 0$. Applying (3.22), (3.25), (3.28), Lemma 2.10, the condition that $f$ and $g$ share $0,1, \infty \mathrm{IM}$, and the supposition that $f$ is not a quasi-Möbius transformation of $g$, we get

$$
\begin{aligned}
\bar{N}_{(1,2)}(r, f, a, b) & \leq N_{1)}(r, 1 /(f-a))+S(r, f) \\
& \leq \bar{N}\left(r, 1 /\left\{b^{\prime}(b-a)\right\}\right)+S(r, f)=S(r, f),
\end{aligned}
$$

which together with (3.28) implies (2.46). Again from (3.17), (3.22) and the condition that $f$ and $g$ share the set $\{a, b\}$ IM we get

$$
\bar{N}(r, 1 /(f-a))+\bar{N}(r, 1 /(g-b))-2 \bar{N}_{0}(r, a, b)=S(r, f) ;
$$

this together with (2.46) and Lemma 2.12 implies that $f$ is a quasi-Möbius transformation of $g$, which contradicts the above supposition. Thus $b^{\prime}=0$, and so $b$ is a constant. Noting that $a \neq b$ and that $f$ and $g$ share $0,1, \infty$ CM, from (3.22), (3.23) and (i)-(iii) of Lemma 2.5, we get

$$
\begin{aligned}
& g-b=0 \Rightarrow f-a=0, \\
& f-b=0 \Rightarrow g-a=0 .
\end{aligned}
$$

From (3.29), (3.30) and (3.25) and the assumptions of Theorem 1.1 we get

$$
N_{1)}(r, 1 /(g-b))+N_{1)}(r, 1 /(f-b))=0 .
$$

From (3.31) and Lemma 2.11 we see that $f$ and $g$ are given by one of the nine expressions in Lemma 2.11. Suppose that $f$ and $g$ have the form (i) of Lemma 2.11. Then

$$
f=\frac{e^{3 \gamma}-1}{e^{\gamma}-1}, \quad g=\frac{e^{-3 \gamma}-1}{e^{-\gamma}-1},
$$

with $b=3 / 4$. From (3.26)-(3.28) and (3.32) we get $a=3$, and so we obtain the conclusion II(i) of Theorem 1.1. Suppose that $f$ and $g$ have one of the forms (ii)-(ix) in Lemma 2.11. As above we obtain the conclusions II(ii)-(vi) of Theorem 1.1, where $a=3 / 2$ and $b=-3 ; a=1 / 3$ and $b=4 / 3 ; a=2 / 3$ 
and $b=-1 / 3 ; a=-2$ and $b=1 / 4 ;$ and $a=-1 / 2$ and $b=4$, in the cases II(ii)-(vi) of Theorem 1.1 respectively.

SubCASE 2.2. Suppose that (3.2) and

$$
\bar{N}_{0}(r, a)=S(r, f) .
$$

Proceeding as in the proof of Subcase 2.1, we get the conclusions II(i)-(vi) of Theorem 1.1, where $a=3 / 4$ and $b=3 ; a=-3$ and $b=3 / 2 ; a=4 / 3$ and $b=1 / 3 ; a=-1 / 3$ and $b=2 / 3 ; a=1 / 4$ and $b=-2$; and $a=4$ and $b=-1 / 2$, in the cases II(i)-(vi) of Theorem 1.1 respectively.

SubCASE 2.3. Suppose that (3.3) and (3.18) hold. Proceeding as at the beginning of Subcase 2.1 we see that $f$ and $g$ share $0,1, \infty \mathrm{CM}$, and that $f, g$ are given by one of the three expressions (i)-(iii) of Lemma 2.5. Applying (3.3), Lemmas 2.6, 2.7, 2.9, 2.10, the condition that $f$ and $g$ share the set $\{a, b\} \mathrm{IM}$, and the supposition that $f$ is not a quasi-Möbius transformation of $g$, we get

$$
\begin{aligned}
T(r, f)-T(r, g) & =N_{2)}(r, 1 /(f-a))-N_{2)}(r, 1 /(g-b))+S(r, f) \\
& =\bar{N}_{(2,1)}(r, a, b)-\bar{N}_{(1,2)}(r, a, b)+S(r, f)=S(r, f),
\end{aligned}
$$

SO

$$
\bar{N}_{(2,1)}(r, a, b)-\bar{N}_{(1,2)}(r, a, b)=S(r, f) .
$$

If (2.46) holds, from Lemma 2.12 we see that $f$ is a quasi-Möbius transformation of $g$, this contradicts the above supposition. Thus $\bar{N}_{(2,1)}(r, a, b)+$ $\bar{N}_{(1,2)}(r, a, b) \neq S(r, f)$. Applying (3.34) we get

$$
\begin{aligned}
& \bar{N}_{(2,1)}(r, a, b) \neq S(r, f), \\
& \bar{N}_{(1,2)}(r, a, b) \neq S(r, f) .
\end{aligned}
$$

From (3.8)-(3.11) and (3.13)-(3.14) we get

$$
\begin{aligned}
& f-a=\frac{h_{1}-a h_{0}+a-1}{h_{0}-1}, \\
& g-b=\frac{h_{1}^{-1}-b h_{0}^{-1}+b-1}{h_{0}^{-1}-1}
\end{aligned}
$$

and

$$
T(r, g)+T\left(r, h_{1}\right)+T\left(r, h_{0}\right)=O(T(r, f)) \quad(r \notin E) .
$$

From (3.11), (3.12) and (3.39) we get

$$
T(r, \alpha)+T(r, \beta)=S(r, f) ;
$$

here and below,

$$
\alpha=\frac{h_{1}^{\prime}}{h_{1}} \quad \text { and } \quad \beta=\frac{h_{0}^{\prime}}{h_{0}}
$$


From the supposition that $f$ is not a quasi-Möbius transformation of $g$, we get $a \beta-a \alpha+a^{\prime} \not \equiv 0$. Let $z_{0}$ be a zero of $f-a$ with multiplicity 2 , and of $g-b$ with multiplicity 1 , such that $z_{0} \notin S_{1}$, where

$$
\begin{aligned}
S_{1}= & \{z: \alpha(z)=0, \infty\} \cup\{z: \beta(z)=0, \infty\} \\
& \cup\{z: \beta(z)-\alpha(z)=0, \infty\} .
\end{aligned}
$$

From (3.37) and (3.42) we get

$$
h_{1}\left(z_{0}\right)-a\left(z_{0}\right) h_{0}\left(z_{0}\right)+a\left(z_{0}\right)-1=0
$$

and

$$
h_{1}\left(z_{0}\right) \alpha\left(z_{0}\right)-h_{0}\left(z_{0}\right)\left[a^{\prime}\left(z_{0}\right)+a\left(z_{0}\right) \cdot \beta\left(z_{0}\right)\right]+a^{\prime}\left(z_{0}\right)=0 .
$$

From (3.43) and (3.44) we get

$$
\begin{aligned}
& h_{1}\left(z_{0}\right)=\frac{\left\{a\left(z_{0}\right)-a^{2}\left(z_{0}\right)\right\} \beta\left(z_{0}\right)+a^{\prime}\left(z_{0}\right)}{a\left(z_{0}\right) \beta\left(z_{0}\right)-a\left(z_{0}\right) \alpha\left(z_{0}\right)+a^{\prime}\left(z_{0}\right)}, \\
& h_{0}\left(z_{0}\right)=\frac{a^{\prime}\left(z_{0}\right)+\left\{1-a\left(z_{0}\right)\right\} \alpha\left(z_{0}\right)}{a\left(z_{0}\right) \beta\left(z_{0}\right)-a\left(z_{0}\right) \alpha\left(z_{0}\right)+a^{\prime}\left(z_{0}\right)} .
\end{aligned}
$$

Let

$$
f_{1}=\frac{\left(a \beta-a \alpha+a^{\prime}\right) h_{1}}{\left(a-a^{2}\right) \beta+a^{\prime}}, \quad f_{2}=\frac{\left(a \beta-a \alpha+a^{\prime}\right) h_{0}}{a^{\prime}+(1-a) \alpha},
$$

$$
T(r)=T\left(r, f_{1}\right)+T\left(r, f_{2}\right), \quad S(r)=o(T(r)) \quad(r \rightarrow \infty, r \notin E) .
$$

From (3.8)-(3.11), (3.41), (3.47) and (3.48) we get

$$
S(r)=S(r, f) \text {. }
$$

On the other hand, from (3.8), (3.12), (3.40), (3.47)-(3.49) we have

$$
\bar{N}\left(r, f_{j}\right)+\bar{N}\left(r, 1 / f_{j}\right)=S(r) \quad(j=1,2) .
$$

From (3.45)-(3.47) we have $f_{1}\left(z_{0}\right)=f_{2}\left(z_{0}\right)=1$, and so

$$
\bar{N}_{(2,1)}(r, a, b) \leq N_{0}\left(r, 1 ; f_{1}, f_{2}\right)+S(r) .
$$

From (3.35), (3.49) and (3.51) we have

$$
N_{0}\left(r, 1 ; f_{1}, f_{2}\right) \neq S(r) .
$$

From $(3.47),(3.48),(3.50),(3.52)$ and Lemma 2.4 we know that there exist two integers $s$ and $t(|s|+|t|>0)$ such that

$$
f_{1}^{s} \cdot f_{2}^{t}=1 \text {. }
$$

From (3.8)-(3.10), (3.40), (3.47), (3.53) and Lemma 2.7 we get

$$
T(r, f)=T(r, g)+S(r, f) .
$$

On the other hand, from (3.9)-(3.11) we have

$$
\frac{h_{0}\left(z_{0}\right)}{h_{1}\left(z_{0}\right)}=\frac{b\left(z_{0}\right)}{a\left(z_{0}\right)}, \quad \frac{1}{h_{1}\left(z_{0}\right)}=\frac{b\left(z_{0}\right)-1}{a\left(z_{0}\right)-1} .
$$


Now (3.44) can be rewritten as

$$
\alpha\left(z_{0}\right)-\frac{h_{0}\left(z_{0}\right)}{h_{1}\left(z_{0}\right)}\left[a^{\prime}\left(z_{0}\right)+a\left(z_{0}\right) \cdot \beta\left(z_{0}\right)\right]+\frac{a^{\prime}\left(z_{0}\right)}{h_{1}\left(z_{0}\right)}=0 .
$$

From (3.55) and (3.56) we get

$$
\alpha\left(z_{0}\right)-\frac{b\left(z_{0}\right)}{a\left(z_{0}\right)} \cdot\left[a^{\prime}\left(z_{0}\right)+a\left(z_{0}\right) \cdot \beta\left(z_{0}\right)\right]+a^{\prime}\left(z_{0}\right) \cdot \frac{b\left(z_{0}\right)-1}{a\left(z_{0}\right)-1}=0 .
$$

From (3.35) and (3.57) we get

$$
\alpha-\frac{b\left(a^{\prime}+a \cdot \beta\right)}{a}+\frac{(b-1) a^{\prime}}{a-1}=0 .
$$

Similarly, from (3.36), (3.38), in the same manner as above we deduce

$$
-\alpha-\frac{a\left(b^{\prime}-b \beta\right)}{b}+\frac{(a-1) b^{\prime}}{b-1}=0 .
$$

Again from (3.3), (i)-(iii) of Lemmas 2.5-2.7 and Lemmas 2.9-2.10, and from the condition that $f$ and $g$ share the set $\{a, b\}$ IM, we deduce

$$
\begin{aligned}
T(r, f)-T(r, g) & =N_{2)}(r, 1 /(f-b))-N_{2)}(r, 1 /(g-a))+S(r, f) \\
& =\bar{N}_{(2,1)}(r, b, a)-\bar{N}_{(1,2)}(r, b, a)=S(r, f),
\end{aligned}
$$

so

$$
\bar{N}_{(2,1)}(r, b, a)-\bar{N}_{(1,2)}(r, b, a)=S(r, f) .
$$

If $\bar{N}_{(2,1)}(r, b, a)+\bar{N}_{(1,2)}(r, b, a)=S(r, f)$, from Lemma 2.12 we see that $f$ is a quasi-Möbius transformation of $g$, which contradicts the above supposition. Thus $\bar{N}_{(2,1)}(r, b, a)+\bar{N}_{(1,2)}(r, b, a) \neq S(r, f)$. From this and (3.60) we get

$$
\bar{N}_{(2,1)}(r, b, a) \neq S(r, f) \quad \text { and } \quad \bar{N}_{(1,2)}(r, b, a) \neq S(r, f) .
$$

Proceeding as in the proof of (3.58) and (3.59), from (3.61) we get

$$
\begin{gathered}
\alpha-\frac{a\left(b^{\prime}+b \cdot \beta\right)}{b}+\frac{(a-1) b^{\prime}}{b-1}=0, \\
\alpha-\frac{b\left(a^{\prime}-a \beta\right)}{a}+\frac{(b-1) a^{\prime}}{a-1}=0 .
\end{gathered}
$$

From (3.58) and (3.63) we get $a^{\prime}=0$. Similarly, from (3.59) and (3.62) we get $b^{\prime}=0$. Applying (3.58) and (3.59) we have $\alpha-b \beta=0$ and $\alpha-a \beta=0$. Thus from (3.41) and $a \neq b$ we get $\beta=h_{0}^{\prime} / h_{0}=0$, which implies that $h_{0}^{\prime}=0$, and so $h_{0}=c_{1}$, where $c_{1}(\neq 0)$ is a finite complex number. Applying (3.9)-(3.11) we see that $f$ is a Möbius transformation of $g$, which contradicts the above supposition.

SubCASE 2.4. Suppose that

$$
N_{0}(r)=S(r, f) .
$$


Then from (3.64) we get (3.3). From (3.3) and the condition that $f$ and $g$ share the set $\{a, b\}$ IM we have

$$
\begin{aligned}
\bar{N}(r, 1 /(f-a))-\bar{N}_{0}(r, a, b) & =S(r, f), \\
\bar{N}(r, 1 /(f-b))-\bar{N}_{0}(r, b, a) & =S(r, f) .
\end{aligned}
$$

From Lemma 2.3 we see that $f$ and $g$ share 0,1 and $\infty \mathrm{CM}^{*}$. Applying (3.65), Lemmas 2.9, 2.10, and the condition that $f$ and $g$ share the set $\{a, b\}$ IM, we get

$$
T(r, f)-T(r, g)=N_{2)}\left(r, \frac{1}{f-a}\right)-N_{2)}\left(r, \frac{1}{g-b}\right)+S(r, f),
$$

and

$$
\begin{aligned}
N_{2)}(r, 1 /(f-a))- & N_{2)}(r, 1 /(g-b)) \\
& =\bar{N}_{(2,1)}(r, a, b)-\bar{N}_{(1,2)}(r, a, b)+S(r, f) .
\end{aligned}
$$

If (2.46) holds, then from (3.68) and Lemma 2.12 we see that $f$ is a quasiMöbius transformation of $g$, which contradicts the above supposition. Thus

$$
\bar{N}_{(2,1)}(r, a, b)+\bar{N}_{(1,2)}(r, a, b) \neq S(r, f) .
$$

Proceeding as in Subcase 2.3 we have (3.37)-(3.54). From (3.54), (3.67) and (3.68) we have (3.34)-(3.36). In the same manner as in Subcase 2.3 we have (3.58). Similarly, from (3.54), (3.66) and in the same manner as in Subcase 2.3 we get (3.59)-(3.63). From (3.58), (3.59), (3.62) and (3.63) we get $a^{\prime}=b^{\prime}=0$, which reveals that $a$ and $b$ are two distinct finite complex numbers. Moreover, from (3.58) and (3.59) we have $\alpha-b \beta=0$ and $\alpha-a \beta=$ 0 . Applying (3.41) and $a \neq b$ we get $\beta=h_{0}^{\prime} / h_{0}=0$, which implies that $h_{0}^{\prime}=0$, and so $h_{0}=c_{1}$, where $c_{1}(\neq 0)$ is a finite complex number. Applying (3.9)-(3.11) we see that $f$ is a Möbius transformation of $g$. This contradicts the above supposition.

Theorem 1.1 is thus completely proved.

Proof of Theorem 1.2. We discuss the following two cases.

CASE 1. Suppose that $f$ is a quasi-Möbius transformation of $g$. Then from the condition that $f=a \Rightarrow g=b$ we see that $f-a$ and $g-b$ share $0 \mathrm{CM}^{*}$. Noting that $f$ and $g$ are entire functions, from Lemma 2.1 we see that $f$ and $g$ satisfy one of the relations (i), (ii) and (vi) of Lemma 2.1. Proceeding as in Case 1 of the proof of Theorem 1.1 we get I(i), I(iii), I(xii) and $\mathrm{I}(\mathrm{xv})$ of Theorem 1.1.

CASE 2. Suppose that $f$ is not a quasi-Möbius transformation of $g$. Proceeding as in Case 2 in the proof of Theorem 1.1 we get (3.8)-(3.16). From the condition that $a \not \equiv b$ and $f=a \Rightarrow g=b$, we get (3.33). We discuss the following three subcases. 
SubCASE 2.1. Suppose that (3.2) holds. Noting that $f$ and $g$ are entire functions, from (3.2), (3.33), in the same manner as in Subcase 2.2 of the proof of Theorem 1.1 we get II(i) of Theorem 1.1 with $a=3 / 4$ and $b=3$, and get $\mathrm{II}(\mathrm{v})$ of Theorem 1.1 with $a=1 / 4$ and $b=-2$.

SubCASE 2.2. Suppose that (3.17) and (3.18) hold. From (3.17) and (3.33) we get (3.3). Noting that $f$ and $g$ are entire functions, from (3.3), (3.18), in the same manner as in Subcase 2.3 of the proof of Theorem 1.1 we get a contradiction.

SubCASE 2.3. Suppose that (3.64) holds. From (3.16) and (3.64) we get

$$
\bar{N}_{0}\left(r, 1 ; h_{1}, h_{0}\right)=S(r, f) .
$$

From (3.8)-(3.12), (3.69) and the second fundamental theorem we deduce

$$
T\left(r, h_{0}\right)=S(r, f) \text {. }
$$

From (3.13), (3.14) and (3.70) we get (3.54). From (3.54), Lemmas 2.9, 2.10 and the condition that $f=a \Rightarrow g=b$ we get (3.34), (3.67) and (3.68). Next in the same manner as in Subcase 2.4 of the proof of Theorem 1.1 we get contradictions.

Theorem 1.2 is thus completely proved.

Acknowledgements. The authors wish to express their thanks to the anonymous referee for his valuable suggestions and comments.

This project was supported by the NSFC (No. A0324617), the RFDP (No. 20060422049), the NSFC (No. 10771121) and the NSF of Shandong Province, China (No. Z2008A01).

\section{References}

[1] T. C. Alzahary and H. X. Yi, Weighted sharing three values and uniqueness of meromorphic functions, J. Math. Anal. Appl. 295 (2004), 247-257.

[2] - - - Meromorphic functions that weighted sharing three values and one pair, Kodai Math. J. 29 (2006), 13-30.

[3] G. Brosch, Eindeutigkeitssätze für meromorphe Funktionen, Thesis, Technical Univ. of Aachen, 1989.

[4] F. Gross, On the distribution of values of meromorphic functions, Trans. Amer. Math. Soc. 131 (1968), 199-214.

[5] G. G. Gundersen, Meromorphic functions that share three or four values, J. London Math. Soc. 20 (1979), 457-466.

[6] W. K. Hayman, Meromorphic Functions, Clarendon Press, Oxford, 1964.

[7] - Weighted sharing of three values and uniqueness of meromorphic functions, Kodai Math. J. 24 (2001), 421-435.

[8] I. Lahiri, Weighted sharing and uniqueness of meromorphic functions, Nagoya Math. J. 161 (2001), 193-206.

[9] I. Lahiri and P. Sahoo, On a result of G. Brosch, J. Math. Anal. Appl. 331 (2007), $532-546$. 
[10] I. Laine, Nevanlinna Theory and Complex Differential Equations, de Gruyter, Berlin, 1993.

[11] P. Li and C. C. Yang, On two meromorphic functions that share pairs of small functions, Complex Variables Theory Appl. 32 (1997), 177-190.

[12] X. M. Li and H. X. Yi, Meromorphic functions sharing three values, J. Math. Soc. Japan 56 (2004), 147-167.

[13] - - - Uniqueness of meromorphic functions whose derivatives share four small functions, J. Math. Anal. Appl. 352 (2009), 573-582.

[14] A. Z. Mokhon'ko, On the Nevanlinna characteristics of some meromorphic functions, in: Theory of Functions, Functional Analysis and Their Applications, Vol. 14, Izd-vo Khar'kovsk. Un-ta, 1971, 83-87.

[15] C. C. Yang and H. X. Yi, Uniqueness Theory of Meromorphic Functions, Kluwer, Dordrecht, 2003.

[16] H. X. Yi, Uniqueness theorems for meromorphic functions concerning small functions, Indian J. Pure Appl. Math. 32 (2001), 903-914.

[17] - Meromorphic functions with weighted sharing of three values, Complex Variables Theory Appl. 50 (2005), 923-934.

[18] Q. C. Zhang, Meromorphic functions sharing three values, Indian J. Pure Appl. Math. 30 (1999), 667-682.

Department of Mathematics

Ocean University of China

Qingdao, Shandong 266071

People's Republic of China

E-mail: xmli01267@gmail.com
Department of Mathematics Shandong University Jinan, Shandong 250100 People's Republic of China E-mail: hxyi@sdu.edu.cn

Received 31.5.2008

and in final form 21.12.2008 
